TRANSACTIONS OF THE

AMERICAN MATHEMATICAL SOCIETY

Volume 356, Number 10, Pages 4161-4180

S 0002-9947(04)03471-3

Article electronically published on April 16, 2004

\title{
DUAL RADON TRANSFORMS ON AFFINE GRASSMANN MANIFOLDS
}

\author{
FULTON B. GONZALEZ AND TOMOYUKI KAKEHI
}

\begin{abstract}
Fix $0 \leq p<q \leq n-1$, and let $G(p, n)$ and $G(q, n)$ denote the affine Grassmann manifolds of $p$ - and $q$-planes in $\mathbb{R}^{n}$. We investigate the Radon transform $\mathcal{R}^{(q, p)}: C^{\infty}(G(q, n)) \rightarrow C^{\infty}(G(p, n))$ associated with the inclusion incidence relation. For the generic case $\operatorname{dim} G(q, n)<\operatorname{dim} G(p, n)$ and $p+q>$ $n$, we will show that the range of this transform is given by smooth functions on $G(p, n)$ annihilated by a system of Pfaffian type differential operators. We also study aspects of the exceptional case $p+q=n$.
\end{abstract}

\section{INTRODUCTION}

In this paper, we continue our investigation of Radon transforms on affine Grassmann manifolds begun in our previous paper [GK]. One of the ways in which the classical $k$-plane transform and its dual on $\mathbb{R}^{n}$ can be generalized is by considering integral transforms on the Grassmannians $G(k, n)$ of unoriented affine $k$-planes on $\mathbb{R}^{n}$ with respect to the inclusion incidence relation. As well, the integral transforms which are studied here and in [GK] can be considered as an affine analogue of the Radon transforms on compact Grassmannians considered in Grinberg Gr, Grinberg and Rubin GrRu, and Kakehi [K].

Specifically, let us assume that $0 \leq p<q \leq n-1$. Fix a $p$-plane $\ell_{0}$ and a $q$-plane $\xi_{0}$ on $\mathbb{R}^{n}$ such that $\ell_{0} \subset \xi_{0}$, and let $H_{p}$ and $H_{q}$ be the subgroups of the Euclidean motion group $M(n)$ fixing $\ell_{0}$ and $\xi_{0}$, respectively. Then $G(p, n)=M(n) / H_{p}$ and $G(q, n)=M(n) / H_{q}$ are homogeneous spaces in duality in the sense defined by Helgason [H4]. Under this duality, elements $\ell \in G(p, n)$ and $\xi \in G(q, n)$ are incident if $\ell \subset \xi$. Let $\mathcal{R}^{(p, q)}: C_{c}^{\infty}(G(p, n)) \longrightarrow C^{\infty}(G(q, n))$ and $\mathcal{R}^{(q, p)}: C^{\infty}(G(q, n)) \longrightarrow$ $C^{\infty}(G(p, n))$ be the corresponding integral transforms.

In our previous paper, we examined the transform $\mathcal{R}^{(p, q)}$. When $p=0$, this transform reduces to the classical $q$-plane transform on $\mathbb{R}^{n}$. We obtained an explicit inversion formula (GK, Theorem 6.4) and a range characterization theorem (GK, Theorem 7.7) for the Radon transform $\mathcal{R}^{(p, q)}$ on a naturally defined Schwartz class $\mathcal{S}(G(p, n))$. Both theorems rely on an extension of the "projection-slice" theorem to affine Grassmannians, with respect to taking Fourier transforms on their fibers. Our results thus generalize the main range and inversion theorems in Richter Ri], Gonzalez G3] and Helgason [H1].

Received by the editors November 26, 2002 and, in revised form, May 1, 2003 and July 17, 2003.

2000 Mathematics Subject Classification. Primary 44A12; Secondary 43A85.

Key words and phrases. Radon transform, Grassmannian, Pfaffian systems.

(C)2004 American Mathematical Society 
Along the way we derived some useful facts about the algebras of invariant differential operators on both affine and compact Grassmannians. (These are restated in Theorems 3.1 and 4.1 below.) In both cases, these algebras are generated by Pfaffian-type operators; these operators are used in the inversion and range characterization of $\mathcal{R}^{(p, q)}$.

In the present paper, we examine the dual transform $\mathcal{R}^{(q, p)}$. When $\operatorname{dim} G(q, n)<$ $\operatorname{dim} G(p, n)$ and $p+q>n$, we obtain a range characterization in terms of Pfaffian operators similar to the ones mentioned above with some significant differences. (See Remark 3 after Theorem 5.1) The case when $p+q=n$ presents some interesting technical issues, and we treat this case in Section 5.

\section{Preliminaries}

Let $G$ be a Lie group with Lie algebra $\mathfrak{g}$. Denote the universal enveloping algebra of $\mathfrak{g}$ by $\mathfrak{u}(\mathfrak{g})$, and let $\mathfrak{z}(\mathfrak{g})$ denote the subalgebra of $A d(G)$-invariant elements in $\mathfrak{u}(\mathfrak{g})$. We call $\mathfrak{z}(\mathfrak{g})$ the Casimir algebra of $\mathfrak{g}$. If $\lambda$ is any representation of $G$ on a topological vector space $V$, we let $d \lambda$ denote the associated representation of $\mathfrak{u}(\mathfrak{g})$ on the subspace $V^{\infty}$ of smooth vectors in $V$. As an example, suppose that $(g, x) \rightarrow g \cdot x$ is a smooth $G$-action on a manifold $M$. Let $\lambda$ be the associated left regular representation of $G$ on $V=C^{\infty}(M)$. If $X \in \mathfrak{g}, d \lambda(X)$ is the vector field tangent to the curve $t \rightarrow \exp (-t X) \cdot x$. For $X_{1}, \cdots, X_{m} \in \mathfrak{g}$, we then have

$$
\begin{aligned}
& d \lambda\left(X_{1} \cdots X_{m}\right) f(x) \\
& \quad=\left\{\frac{\partial^{m}}{\partial t_{1} \cdots \partial t_{m}} f\left(\exp \left(-t_{m} X_{m}\right) \cdots \exp \left(-t_{1} X_{1}\right) \cdot x\right)\right\}_{t_{1}=\cdots=t_{m}=0} .
\end{aligned}
$$

Since $\lambda(g) \circ d \lambda(U) \circ \lambda\left(g^{-1}\right)=d \lambda(A d(g) U)$ for all $g \in G$ and $U \in \mathfrak{u}(\mathfrak{g})$, we see that $d \lambda(\mathfrak{z}(\mathfrak{g}))$ consists of $G$-invariant differential operators on $M$.

Suppose that $\lambda$ and $\nu$ are representations of $G$ on topological vector spaces $V$ and $W$, respectively. Let $R: V \longrightarrow W$ be a continuous linear map intertwining $\lambda$ and $\nu$. If $V^{\infty}$ and $W^{\infty}$ denote the subspaces of $C^{\infty}$ vectors in $V$ and $W$, respectively, then $R\left(V^{\infty}\right) \subset W^{\infty}$ and $R(d \lambda(U) v)=d \nu(U) R v$ for all $U \in \mathfrak{u}(\mathfrak{g}), v \in V^{\infty}$. In other words, the diagram below commutes:

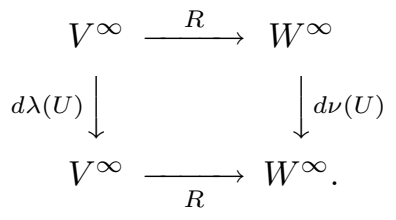

In particular, if $R$ is injective, then, $\operatorname{ker}(d \nu) \subset \operatorname{ker}(d \lambda)$. In case $\operatorname{ker}(d \nu) \varsubsetneqq \operatorname{ker}(d \lambda)$, we have necessary conditions satisfied by the range $R\left(V^{\infty}\right): d \nu(U) R v=0$ for all $v \in V^{\infty}, U \in \operatorname{ker}(d \lambda) \backslash \operatorname{ker}(d \nu)$.

In this paper, $\lambda$ and $\nu$ will denote the left regular representations of a given Lie group $G$ on a various smooth function spaces on homogeneous manifolds of $G$. More specifically, we will consider homogeneous spaces in duality, as follows. Let $H$ and $K$ be closed subgroups for $G$ satisfying the conditions. (See [H2], page 140.)

(i) $G, H, K$, and $H \cap K$ are all unimodular.

(ii) $H K$ is closed in $G$.

(iii) $H \cap K=\left\{h \in H \mid h K \cup h^{-1} K \subset K H\right\}=\left\{k \in K \mid k H \cup k^{-1} H \subset H K\right\}$. 
The coset spaces $X=G / K$ and $\Xi=G / H$ are then said to be homogeneous in duality, and we have the following double fibration

$$
X=G / K \swarrow^{G /(H \cap K)} \searrow \quad G / H=\Xi .
$$

The corresponding Radon transform $R: C_{c}^{\infty}(X) \longrightarrow C^{\infty}(\Xi)$ and its dual Radon transform ${ }^{t} R: C_{c}^{\infty}(\Xi) \longrightarrow C^{\infty}(X)$ given respectively by

$$
\begin{aligned}
R f(\gamma H) & =\int_{H / H \cap K} f(\gamma h K) d h_{H \cap K}, \\
{ }^{t} R \varphi(g K) & =\int_{K / H \cap K} f(g k H) d k_{H \cap K} .
\end{aligned}
$$

Elements $x=g K \in X$ and $\xi=\gamma H \in \Xi$ are said to be incident if, as cosets of $G$, they have a nonempty intersection. The set of all $x \in X$ incident to $\xi$ is easily seen to be $\widehat{\xi}=\{\gamma h K \mid h \in H\}$; similarly, the set of all $\xi \in \Xi$ incident to $x$ is $\check{x}=\{g k H \mid k \in K\}$. The integral for $R f(\xi)$ is thus taken over $\widehat{\xi}$ and that for ${ }^{t} R \varphi(x)$ is taken over $\check{x}$.

For any $g \in G$, let $\tau(g)$ denote the left translation by $g$ on $X$ and $\Xi$, so that $\tau(g) x=g \cdot x, \tau(g) \xi=g \cdot \xi$. The translate $f^{\tau(g)}$ of a function $f$ on $X$ is then given by $f^{\tau(g)}(x)=f\left(g^{-1} \cdot x\right)$. For a function $\varphi$ on $\Xi$, we define $\varphi^{\tau(g)}$ similarly. If $D$ is a differential operator on $X$, its translate $D^{\tau(g)}$ is given by $D^{\tau(g)} f=\left(D f^{\tau\left(g^{-1}\right)}\right)^{\tau(g)}$.

Let $\lambda$ and $\nu$ denote the left regular representations of $G$ on $C^{\infty}(X)$ and $C^{\infty}(\Xi)$, respectively, so that $\lambda(g) f=f^{\tau(g)}, \nu(g) \varphi=\varphi^{\tau(g)}$. The integral for $R$ and ${ }^{t} R$ above show that these transforms intertwine $\lambda$ and $\nu$. Thus $R(d \lambda(U) f)=d \nu(U) R f$ for all $f \in C_{c}^{\infty}(X)$; as stated before, if $R$ is injective, then $\operatorname{ker}(d \nu) \subset \operatorname{ker}(d \lambda)$ and we obtain differential equations satisfied by the range of $R$ in case $\operatorname{ker}(d \nu) \varsubsetneqq \operatorname{ker}(d \lambda)$. It turns out that these equations are often sufficient to characterize the range as well.

If $X$ is a homogeneous space of a compact Lie group, then any integral on $X$ will be taken with respect to the unique normalized $G$-invariant measure on $X$.

We would like to thank the referee for providing valuable suggestions for improving the manuscript.

\section{Pfaffian algebra and Radon transforms ON COMPACT GRASSMANN MANIFOLDS}

Fix an integer $n, n \geq 3$. For any integer $r$ in $\{1,2, \cdots, n\}$, let $P_{r}$ denote the set of all permutations of $\{1,2, \cdots, n\}$ taken $r$ at a time, and let $T_{r}$ denote the collection of all increasing sequences $J=\left\{j_{1}, j_{2}, \cdots, j_{r}\right\}$ of length $r$ in $\{1,2, \cdots, n\}$. Then, of course, $T_{r} \subset P_{r}$. For $J \in T_{r}$, let $\mathfrak{S}(J)$ denote the set of all permutations of $J$ (written as ordered $r$-tuples), so that $P_{r}=\bigcup_{J \in T_{r}} \mathfrak{S}(J)$. If $J^{\prime} \in \mathfrak{S}(J)$, we denote its sign by $\epsilon\left(J^{\prime}\right)$. Let $A=\left(a_{i j}\right)$ be any $n \times n$ matrix with entries in some ring. If $I=\left\{i_{1}, i_{2}, \cdots, i_{r}\right\}$ and $J=\left\{j_{1}, j_{2}, \cdots, j_{r}\right\}$ belong to $T_{r}$, we let $A_{I J}$ denote the $r \times r$ submatrix $\left(a_{i_{k}} j_{l}\right)_{1 \leq k, l \leq r}$. 
The Lie algebra $s o(n)$ of the special orthogonal group $S O(n)$ has basis given by $X_{i j}=E_{i j}-E_{j i}(1 \leq i<j \leq n)$. Let $X$ be the $n \times n$ matrix with vector entries

$$
X=\left(\begin{array}{cccc}
0 & X_{12} & \cdots & X_{1 n} \\
-X_{12} & 0 & \cdots & X_{2 n} \\
& \cdots & \ddots & \\
-X_{1 n} & -X_{2 n} & \cdots & 0
\end{array}\right) \text {. }
$$

Fix an integer $k, 0 \leq k \leq\left[\frac{n}{2}\right]$, and let $I \in T_{2 k}, I=\left\{i_{1}, i_{2}, \cdots, i_{2 k}\right\}$. Let $X_{I}$ denote the $2 k \times 2 k$ submatrix of $X$ given by

$$
X_{I}=\left(\begin{array}{cccc}
0 & X_{i_{1} i_{2}} & \cdots & X_{i_{1} i_{2 k}} \\
-X_{i_{1} i_{2}} & 0 & \cdots & X_{i_{2} i_{2 k}} \\
& \cdots & \ddots & \\
-X_{i_{1} i_{2 k}} & -X_{i_{2} i_{2 k}} & \cdots & 0
\end{array}\right)
$$

and set

$$
W_{I}=\sum_{\sigma} \epsilon(\sigma) X_{\sigma\left(i_{1}\right) \sigma\left(i_{2}\right)} \cdots X_{\sigma\left(i_{2 k-1}\right) \sigma\left(i_{2 k}\right)} \in \mathfrak{u}(s o(n)),
$$

where the sum is taken over all permutations $\sigma$ of $I$ such that $\sigma\left(i_{1}\right)<\sigma\left(i_{2}\right), \cdots$, $\sigma\left(i_{2 k-1}\right)<\sigma\left(i_{2 k}\right)$ and $\sigma\left(i_{1}\right)<\sigma\left(i_{3}\right)<\cdots<\sigma\left(i_{2 k-1}\right)$. Thus $W_{I}$ equals the Pfaffian $\operatorname{Pf}\left(X_{I}\right)$. Note that the factors in each of the summands in (3.3) commute.

Note that $\operatorname{rank}(S O(n))=\left[\frac{n}{2}\right]$. For each integer $k, 1 \leq k<\left[\frac{n}{2}\right]$, let us set

$$
U_{2 k}=\sum_{I \in T_{2 k}} W_{I}^{2}
$$

in addition, let us set

$$
\begin{aligned}
U_{n-1} & =\sum_{I \in T_{n-1}} W_{I}^{2}, & & \text { if } n \text { is odd, } \\
U_{n} & =W_{\{1,2, \cdots, n\}} & & \text { if } n \text { is even. }
\end{aligned}
$$

Theorem 3.1. The elements $U_{2}, U_{4}, \cdots, U_{2\left[\frac{n}{2}\right]}$ are algebraically independent generators of the Casimir algebra $\mathfrak{z}($ so $(n))$.

(See, for example, [KN], Volume II, Chapter XII, Theorem 2.7, or [GK], Theorem 2.3.)

The compact Grassmann manifolds provide a useful model for homogeneous spaces in duality. Let $1 \leq p \neq q \leq n-1$. Let

$$
G=S O(n), \quad K=S(O(p) \times O(n-p)), \quad H=S(O(q) \times O(n-q)),
$$

so that

$$
X=G / K=G_{p, n} \text { and } \Xi=G / H=G_{q, n}
$$

are the compact Grassmann manifolds of $p$ - and $q$-dimensional subspaces of $\mathbb{R}^{n}$. The associated incidence relation is the usual one of inclusion; the corresponding Radon transform $R=R_{p, q}$ and its dual ${ }^{t} R=R_{q, p}$ are defined on $C^{\infty}(X)$ and on $C^{\infty}(\Xi)$, respectively.

If $\operatorname{rank}\left(G_{p, n}\right) \leq \operatorname{rank}\left(G_{q, n}\right)$, the Radon transform $R_{p, q}$ can be inverted. An explicit inversion formula is given by $[\mathrm{K}]$ in the case $q-p$ is even, and later by $\mathrm{GrRu}$ for any $q>p$ (the case $q<p$ reduces to the case $q>p$ by passing to 
orthogonal complements). See also Petrov $[\mathrm{P}]$. We also have the following range characterization:

Theorem $3.2(\mathrm{GK}]$, Theorem 4.2). Suppose that $l:=\operatorname{rank}\left(G_{p, n}\right)<\operatorname{rank}\left(G_{q, n}\right)$. Then

$$
\begin{aligned}
R_{p, q} C^{\infty}\left(G_{p, n}\right) & =\left\{\varphi \in C^{\infty}\left(G_{q, n}\right) \mid d \nu\left(W_{I}\right) \varphi=0, \text { for all } I \in T_{2 l+2}\right\} \\
& =\left\{\varphi \in C^{\infty}\left(G_{q, n}\right) \mid d \nu\left(U_{l+1}\right) \varphi=0\right\}
\end{aligned}
$$

\section{INVARIANT DIFFERENTIAL OPERATORS AND RADON TRANSFORMS ON AFFINE GRASSMANNIANS}

This paper and its predecessor [GK] address the question of extending the above compact results to analogous transforms on affine $p$ - and $q$-planes in $\mathbb{R}^{n}$. Under the rubric of dual homogeneous spaces, our ambient group $G$ is now the Euclidean motion group $M(n)=O(n) \rtimes \mathbb{R}^{n}$, and $X$ and $\Xi$ are now the affine Grassmann manifolds $G(p, n)$ and $G(q, n)$ of affine $p$ - and $q$-planes, respectively, in $\mathbb{R}^{n}$

We assume throughout this paper that $p<q$.

Set $\ell_{0}=\mathbb{R} \mathbf{e}_{1} \oplus \cdots \oplus \mathbb{R} \mathbf{e}_{p}$ and $\xi_{0}=\mathbb{R} \mathbf{e}_{1} \oplus \cdots \oplus \mathbb{R} \mathbf{e}_{q}$; their stabilizers in $M(n)$ are $H_{p}=M(p) \times O(n-p)$ and $H_{q}=M(q) \times O(n-q)$, respectively. Thus $G(p, n)=$ $M(n) / H_{p}$ and $G(q, n)=M(n) / H_{q}$. Moreover, $\operatorname{dim} G(p, n)=(p+1)(n-p)$ and $\operatorname{dim} G(q, n)=(q+1)(n-q)$. It is easy to show that $M(n), H_{p}$ and $H_{q}$ satisfy conditions (i), (ii), and (iii) in Section 2. The incidence relation is then the usual one of inclusion. We denote the corresponding integral transforms by $\mathcal{R}^{(p, q)}$ and $\mathcal{R}^{(q, p)}\left(={ }^{t} \mathcal{R}^{(p, q)}\right)$ :

$$
\begin{aligned}
& \mathcal{R}^{(p, q)} f(\xi)=\int_{\ell \subset \xi} f(\ell) d \ell, \quad \xi \in G(q, n), \quad f \in C_{c}^{\infty}(G(p, n)), \\
& \mathcal{R}^{(p, q)} \varphi(\ell)=\int_{\xi \supset \ell} \varphi(\xi) d \xi, \quad \ell \in G(p, n), \quad \varphi \in C^{\infty}(G(q, n)) .
\end{aligned}
$$

Here $d \ell$ and $d \xi$ are the canonically defined measures from (2.4) and (2.5) on $\widehat{\xi}=$ $\{\ell \in G(p, n) \mid \ell \subset \xi\}$ and $\check{\ell}=\{\xi \in G(q, n) \mid \xi \supset \ell\}$. Note that the integral in (4.2) takes place over a compact set. In [GK], we undertook a study of the transform $\mathcal{R}^{(p, q)}$, producing both an explicit inversion formula ([GK], Theorem 6.4) and a range characterization (GK], Theorem 7.7).

We now undertake a similar study of the dual transform $\mathcal{R}^{(q, p)}$, focusing on characterizing its range. To this end, we introduce some additional notation and recall some results pertaining to the Casimir algebra of $M(n)$.

We define the rank of the group $M(n)$ to be $r=\left[\frac{n+1}{2}\right]$; likewise we define the rank of $G(p, n)$ (respectively $G(q, n))$ to be $s=\min (p+1, n-p)$ (respectively $m=\min (q+1, n-q))$. It turns out that the Casimir algebra of $M(n)$ is generated by $r$ algebraically independent elements; the first $s$ of these give rise to a generating set for $\mathbb{D}(G(p, n))$, the algebra of $M(n)$-invariant differential operators on $G(p, n)$. (See Theorem 4.1 below.)

Let $\lambda$ and $\nu$ denote the left regular representations of $M(n)$ on smooth functions on $G(p, n)$ and on $G(q, n)$, respectively.

The Lie algebra $\mathfrak{m}(n)=s o(n) \rtimes \mathbb{R}^{n}$ of the Euclidean motion group $M(n)$ has basis consisting of the elementary skew symmetric matrices $X_{i j} \quad(1 \leq i<j \leq n)$, as well as the standard basis vectors $E_{i} \quad(1 \leq i \leq n)$ of $\mathbb{R}^{n}$. Fix an integer $d$ in 
$\left\{1,2, \cdots,\left[\frac{n+1}{2}\right]\right\}$ and let $J \in T_{2 d-1}, J=\left\{j_{1}, j_{2}, \cdots, j_{2 d-1}\right\}$. Define the element $V_{J} \in \mathfrak{u}(\mathfrak{m}(n))$ by

$$
\begin{aligned}
V_{J} & =\operatorname{Pf}\left(\begin{array}{ccccc}
0 & E_{j_{1}} & E_{j_{2}} & \cdots & E_{j_{2 d-1}} \\
-E_{j_{1}} & 0 & X_{j_{1} j_{2}} & \cdots & X_{j_{1} j_{2 d-1}} \\
-E_{j_{2}} & -X_{j_{1} j_{2}} & 0 & \cdots & X_{j_{2} j_{2 d-1}} \\
& \cdots & & \ddots & \\
-E_{j_{2 d-1}} & -X_{j_{1} j_{2 d-1}} & -X_{j_{2} j_{2 d-1}} & \cdots & 0
\end{array}\right) \\
& =\sum_{\sigma} \epsilon(\sigma) E_{\sigma\left(j_{1}\right)} X_{\sigma\left(j_{2}\right) \sigma\left(j_{3}\right)} \cdots X_{\sigma\left(j_{2 d-2}\right)} \sigma\left(j_{2 d-1}\right)
\end{aligned}
$$

where the sum extends over all $\sigma \in \mathfrak{S}(J)$ such that $\sigma\left(j_{2}\right)<\sigma\left(j_{3}\right), \cdots, \sigma\left(j_{2 d-2}\right)<$ $\sigma\left(j_{2 d-1}\right)$ and $\sigma\left(j_{2}\right)<\sigma\left(j_{4}\right)<\cdots<\sigma\left(j_{2 d-2}\right)$. Note that the factors in each of the summands above commute, and we can also write

$$
V_{J}=\sum_{k=1}^{2 d-1}(-1)^{k-1} E_{j_{k}} W_{J \backslash\left\{j_{k}\right\}}=\sum_{j \in J} \epsilon(j, J \backslash\{j\}) E_{j} W_{J \backslash\{j\}},
$$

where $W_{J \backslash\left\{j_{k}\right\}} \in \mathfrak{u}(s o(n))$ is an order $2 d-2$ Pfaffian of the type introduced in Section 2. Next let

$$
Q_{2 d}=\sum_{J \in T_{2 d-1}} V_{J}^{2} \in \mathfrak{u}(\mathfrak{m}(n)) .
$$

Theorem 4.1. $\quad$ (i) $\mathfrak{z}(\mathfrak{m}(n))$ is generated by $Q_{2}, Q_{4}, \cdots, Q_{2\left[\frac{n+1}{2}\right]}$, and these are algebraically independent.

(ii) Let $s=\operatorname{rank}(G(p, n))$. Then the algebra $\mathbb{D}(G(p, n))$ of $M(n)$-invariant differential operators on $G(p, n)$ is generated by the elements $d \lambda\left(Q_{2}\right), d \lambda\left(Q_{4}\right)$, $\cdots, d \lambda\left(Q_{2 s}\right)$, and these are algebraically independent.

The proof of (i) can be found in [G2]; (ii) is in GH].

We note that when $X$ is a Riemannian symmetric space with connected isometry group $G$, then the algebra $\mathbb{D}(X)$ of left $G$-invariant differential operators on $X$ is generated by $\operatorname{rank}(X)$ algebraically independent elements. This justifies our present definition of rank for affine Grassmann manifolds.

Let $\pi_{p}: G(p, n) \rightarrow G_{p, n}$ be the projection mapping of any $p$-plane $\ell$ onto the parallel $p$-plane $\sigma$ through the origin. Then the intersection $\ell \cap \sigma^{\perp}$ consists of a single point $x$. Write $\ell=(\sigma, x)$, so that $G(p, n)=\left\{(\sigma, x) \in G_{p, n} \times \mathbb{R}^{n} \mid \sigma \perp x\right\}$. Similarly, $G(q, n)=\left\{(\eta, y) \in G_{q, n} \times \mathbb{R}^{n} \mid \eta \perp y\right\}$. Keeping in mind that $q>p$, the transform $\mathcal{R}^{(q, p)}$ can now be written in terms of these parametrizations as follows.

If $\ell=(\sigma, x)$, then any $q$-plane incident to $\ell$ is of the form $\left(\eta, P r_{\eta^{\perp}} x\right)$, where $\eta \in G_{q, n}, \eta \supset \sigma$, and $P r_{\eta^{\perp}}$ denotes the orthogonal projection onto the subspace $\eta^{\perp}$. Now the set consisting of such $\eta$ is an orbit of the subgroup $K_{\sigma}$ of $O(n)$ fixing $\sigma$. We let $d_{\sigma} \eta$ denote the normalized $K_{\sigma}$-invariant measure on this orbit. Now the transform $\Psi$ given by

$$
\begin{aligned}
\Psi: C^{\infty}(G(q, n)) & \longrightarrow C^{\infty}(G(p, n)) \\
\varphi & \longmapsto \Psi \varphi(\sigma, x):=\int_{\eta \in G_{q, n} \eta \supset \sigma} \varphi\left(\eta, P r_{\eta^{\perp}} x\right) d_{\sigma} \eta
\end{aligned}
$$


coincides with $\mathcal{R}^{(q, p)}$. Certainly the integral representing $\Psi$ above is invariant under all $g \in M(n)$. This equivariance shows that $\Psi$ coincides with $\mathcal{R}^{(q, p)}$ up to a constant multiple, which we declare to be 1.

\section{RANGe ChaRACTERization of $\mathcal{R}^{(q, p)}$}

Assume that $p<q$ and $\operatorname{rank}(G(p, n))>\operatorname{rank}(G(q, n))$. We will show that the range of $\mathcal{R}^{(q, p)}$ is characterized by a system of Pfaffian type equations. This system cannot be reduced to a single invariant equation, as in the case of the transform $\mathcal{R}^{(p, q)}$, unless possibly $p+q=n$.

Since $\operatorname{rank}(G(p, n))>\operatorname{rank}(G(q, n))$, we must have $q>\left[\frac{n}{2}\right]$. Otherwise, $2 q \leq n$, so $\operatorname{rank}(G(q, n))=\min (q+1, n-q) \geq q \geq p+1 \geq \operatorname{rank}(G(p, n))$, a contradiction. Consequently, $\operatorname{rank}(G(q, n))=n-q$. Let $m$ denote this number. Also the inequality $n-q<p+1$ implies that $p+q \geq n$. This means that we also have $\operatorname{rank}\left(G_{q, n}\right)=$ $n-q \leq \min (p, n-p)=\operatorname{rank}\left(G_{p, n}\right)$, so that the corresponding compact transform $R_{q, p}: C^{\infty}\left(G_{q, n}\right) \rightarrow C^{\infty}\left(G_{p, n}\right)$ is injective.

Now from (4.6), $\mathcal{R}^{(q, p)}$ is given by the following integral:

$$
\mathcal{R}^{(q, p)} \varphi(\sigma, x)=\int_{\eta \supset \sigma} \varphi\left(\eta, P r_{\eta^{\perp}} x\right) d \eta, \quad \varphi \in C^{\infty}(G(q, n)) .
$$

From general considerations, (See [H4] Chapter II, Proposition 2.4.) it follows that $\mathcal{R}^{(q, p)} \varphi \in C^{\infty}(G(p, n))$. Moreover, we have the following lemma.

Lemma 5.1. If $p<q$ and $p+q \geq n$, then $\mathcal{R}^{(q, p)}$ is an injective operator from $C^{\infty}(G(q, n))$ to $C^{\infty}(G(p, n))$.

Proof. For $\ell \in G(p, n)$, let $\ell=(\sigma, x), \sigma \in G_{p, n}, x \in \sigma^{\perp}$. Then any $\xi \in G(q, n)$ including $\ell$ is written as $\xi=\eta+x$ for $\eta \in G_{q, n}$ with $\eta \supset \sigma$. Now let us denote $\varphi_{x}(\eta)=\varphi(\eta+x)$ and regard it as a function on $G_{q, n}$. Here we note that $\mathcal{R}^{(q, p)}$ is $M(n)$-invariant and, in particular, invariant under the translation $\tau(x)$. Thus we have

$$
\left(\mathcal{R}^{(q, p)} \varphi\right)(\sigma, x)=\left(R_{q, p} \varphi_{x}\right)(\sigma)=\int_{\eta \supset \sigma} \varphi_{x}(\eta) d_{\sigma} \eta
$$

If $p<q$ and $p+q \geq n$, then $\operatorname{rank}\left(G_{q, n}\right) \leq \operatorname{rank}\left(G_{p, n}\right)$ and therefore $R_{q, p}$ is injective. Hence, if $\left(\mathcal{R}^{(q, p)} \varphi\right)(\sigma, x) \equiv 0$, then $\varphi_{x}(\eta) \equiv 0$ on $G_{q, n}$ for all $x \in \sigma^{\perp}$. Since $\sigma^{\perp} \supset \eta^{\perp}$, $\varphi(\eta, y)=0$ for all $\eta \in G_{q, n}$ and for all $y \in \eta^{\perp}$, that is, $\varphi \equiv 0$ on $G(q, n)$.

Note that the hypothesis above is equivalent to the condition that $\operatorname{rank}(G(q, n))$ $<\operatorname{rank}(G(p, n))$ and $p<q$. Rubin $\mathrm{Ru} 2$ has obtained an important extension to this lemma as follows. In the case when $\operatorname{rank}(G(q, n))=\operatorname{rank}(G(p, n))$, then $\mathcal{R}^{(q, p)}$ is injective on functions on $G(q, n)$ satisfying mild decay conditions at infinity. This result generalizes the injectivity of the classical dual transform on rapidly decreasing hyperplane functions (See G1] or [So.) This extension is important because $\mathcal{R}^{(q, p)}$ is no longer injective in the absence of these decay conditions when $\operatorname{rank}(G(q, n))=$ $\operatorname{rank}(G(p, n))$, since, in this case, we would have $\operatorname{rank}\left(G_{q, n}\right)>\operatorname{rank}\left(G_{p, n}\right)$.

The theorem below provides the range characterization for $\mathcal{R}^{(q, p)} C^{\infty}(G(q, n))$ in the case when $p+q>n$. 
Theorem 5.1. Assume that $p<q, p+q>n$, and $s=\operatorname{rank}(G(p, n))>m=$ $\operatorname{rank}(G(q, n))$. Then the range $\mathcal{R}^{(q, p)} C^{\infty}(G(q, n))$ consists precisely of those functions $f \in C^{\infty}(G(p, n))$ which satisfy the differential equations $d \lambda\left(V_{J}\right) f=0$ and $d \lambda\left(W_{I}\right) f=0$ for all $J \in T_{2 m+1}, I \in T_{2 m+2}$.

Note that since $m<\operatorname{rank}(G(p, n))$, the differential operators $d \lambda\left(V_{J}\right)$ and $d \lambda\left(W_{I}\right)$ are not all zero on $G(p, n)$.

We first prove that the above equations are necessarily satisfied by the range. Now $\mathcal{R}^{(q, p)}$ is $M(n)$-equivariant. So for any $U \in \mathfrak{u}(\mathfrak{m}(n))$ we have the following commutative diagram:

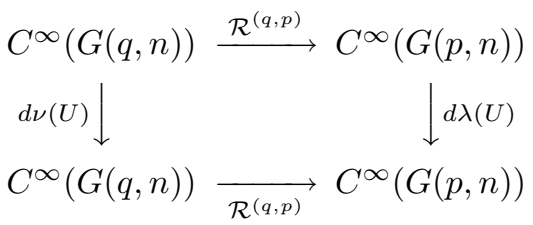

From the considerations given in the preliminaries, it is sufficient to show that the differential operators $d \nu\left(V_{J}\right)$ and $d \nu\left(W_{I}\right)$ vanish on $G(q, n)$.

Let $\varphi \in C^{\infty}(G(q, n))$. Set $\eta_{0}=\mathbb{R} e_{1} \oplus \cdots \oplus \mathbb{R} e_{q}$. We now show that

$$
\left(d \nu\left(V_{J}\right) \varphi\right)\left(\eta_{0}, 0\right)=0
$$

for any $J \in T_{2 m+1}$. Now

$$
V_{J}=\sum \pm X_{j_{1}, j_{2}} \cdots X_{j_{2 m-1}, j_{2 m}} E_{j_{2 m+1}}
$$

where the sum is taken over indices $\left\{j_{1}, j_{2}, \cdots, j_{2 m+1}\right\} \in \mathfrak{S}(J)$. Equation (5.4) will follow if we can show that

$$
\left(d \nu\left(X_{j_{1}, j_{2}} \cdots X_{j_{2 m-1}, j_{2 m}} E_{j_{2 m+1}}\right) \varphi\right)\left(\eta_{0}, 0\right)=0 .
$$

To show (5.6), we essentially count indices. If $j_{2 m+1} \in\{1, \cdots, q\}$, then we write the left-hand side of (5.6) as

$$
d \nu\left(E_{j_{2 m+1}}\right)\left(d \nu\left(X_{j_{1}, j_{2}} \cdots X_{j_{2 m-1}, j_{2 m}}\right) \varphi\right)\left(\eta_{0}, 0\right),
$$

which clearly vanishes. If $j_{2 m+1}>q$, we claim that at least one pair $\left\{j_{2 k-1}, j_{2 k}\right\}$ belongs to $\{1, \cdots, q\}$. If not, then (since $m=n-q$ ), this forces $j_{2 m+1} \leq q$, which was already excluded. We write the left-hand side of (5.6) as

$$
d \nu\left(X_{j_{2 k-1}, j_{2 k}}\right)\left(d \nu\left(X_{j_{1}, j_{2}} \cdots \widehat{X}_{j_{2 k-1}, j_{2 k}} \cdots X_{j_{2 m-1}, j_{2 m}} E_{j_{2 m+1}}\right) \varphi\right)\left(\eta_{0}, 0\right),
$$

which clearly also vanishes. In either case, we have proven (5.6), and thus (5.4).

In the following lemma, we now recall the conjugation rules in [GK], $\S 2$ and $\S 5$ governing the elements $V_{J}$ and $W_{I}$ in $\mathfrak{u}(\mathfrak{m}(n))$.

Lemma 5.2. For $u \in S O(n)$ and $x \in \mathbb{R}^{n}$,

$$
\begin{aligned}
& A d(u) W_{I}=\sum_{L \in T_{2 m+2}} \operatorname{det}\left(u_{L I}\right) W_{L}, \quad \operatorname{Ad}(u) V_{J}=\sum_{R \in T_{2 m+1}} \operatorname{det}\left(u_{R J}\right) V_{R}, \\
& A d(x) W_{I}=W_{I}-\sum_{P \in T_{2 m+1}} e_{P I}(x) V_{P}, \quad A d(x) V_{J}=V_{J},
\end{aligned}
$$

where $u_{L I}=\left(u_{i j}\right)_{i \in I, l \in L}$, and $e_{P I}(x)$ is a scalar which can be explicitly calculated. 
We also recall that Lemma 3.1 in $[\mathrm{GK}$ states that

$$
d \nu\left(W_{I}\right) \varphi(\eta, 0)=0,
$$

for all $\eta \in G_{q, n}$ and for all $I \in T_{2 m+2}$. We now show that for each such $\eta$ and $J \in T_{2 m+1}$,

$$
d \nu\left(V_{J}\right) \varphi(\eta, 0)=0 .
$$

For this, simply choose $u \in S O(n)$ for which $u \cdot \eta_{0}=\eta$. Then the left-hand side of (5.11) equals

$$
\begin{aligned}
\left(d \nu\left(V_{J}\right) \varphi\right)^{\tau\left(u^{-1}\right)}\left(\eta_{0}, 0\right) & =\left(d \nu\left(\operatorname{Ad}\left(u^{-1}\right) V_{J}\right) \varphi^{\tau\left(u^{-1}\right)}\right)\left(\eta_{0}, 0\right) \\
& =\sum_{R} d_{R J}\left(u^{-1}\right)\left(d \nu\left(V_{R}\right) \varphi^{\tau\left(u^{-1}\right)}\right)\left(\eta_{0}, 0\right) \\
& =0
\end{aligned}
$$

using (5.9) and (5.4) (with $\varphi$ replaced by $\left.\varphi^{\tau\left(u^{-1}\right)}\right)$. Finally, let $(\eta, x) \in G(q, n)$ be arbitrary. From (5.9), (5.10), (5.11), we obtain

$$
\begin{aligned}
\left(d \nu\left(W_{I}\right) \varphi\right)(\eta, x) & =\left(d \nu\left(\operatorname{Ad}(-x) W_{I}\right) \varphi^{\tau(-x)}\right)(\eta, 0) \\
& =\left(d \nu\left(W_{I}\right) \varphi^{\tau(-x)}\right)(\eta, 0)-\sum_{P} e_{P I}(-x)\left(d \nu\left(V_{P}\right) \varphi^{\tau(-x)}\right)(\eta, 0) \\
& =0 .
\end{aligned}
$$

Likewise, from (5.9) and (5.11), we have

$$
\left(d \nu\left(V_{J}\right) \varphi\right)(\eta, x)=\left(d \nu\left(V_{J}\right) \varphi^{\tau(-x)}\right)(\eta, 0)=0 .
$$

(In applying (5.11), we replace $\varphi$ by $\varphi^{\tau(-x)}$.)

For the converse, we now show that if $f \in C^{\infty}(G(p, n))$ satisfies the Pfaffian equations $d \lambda\left(W_{I}\right) f=0$, and $d \lambda\left(V_{J}\right) f=0$ for all $I \in T_{2 m+2}, J \in T_{2 m+1}$, then $f=\mathcal{R}^{(q, p)} \varphi$ for some $\varphi \in C^{\infty}(G(q, n))$.

It will be more convenient to move our calculations to the product manifolds $G_{p, n} \times \mathbb{R}^{n}$ and $G_{q, n} \times \mathbb{R}^{n} . M(n)$ acts transitively on $G_{p, n} \times \mathbb{R}^{n}$ via $(u, x) \cdot(\sigma, y)=$ $(u \cdot \sigma, u \cdot y+x)$. The subgroup fixing the point $\left(\sigma_{0}, 0\right)$ (where $\sigma_{0}=\mathbb{R} e_{1} \oplus \cdots \oplus \mathbb{R} e_{p}$ ) is $O(p) \times O(n-p) \subset O(n)$, so that $G_{p, n} \times \mathbb{R}^{n}=M(n) / O(p) \times O(n-p)$. Let $\pi_{p}$ denote the projection of $G_{p, n} \times \mathbb{R}^{n}$ onto $G(p, n)$ given by $\pi_{p}(\sigma, x)=\left(\sigma, \operatorname{Pr}_{\sigma^{\perp}} x\right)$, the $p$-plane through $x$ parallel to $\sigma$. If $h \in C^{\infty}(G(p, n))$, then the function $H=h \circ \pi_{p}$ is a smooth function on $G_{p, n} \times \mathbb{R}^{n}$ satisfying $H(\sigma, x)=H(\sigma, x+v)$ for all $v \in \sigma$. Conversely, by using local cross sections on $M(n)$, for example, it is easy to see that each smooth function $H(\sigma, x)$ on $G_{p, n} \times \mathbb{R}^{n}$ which satisfies $H(\sigma, x)=H(\sigma, x+v)$ for all $v \in \sigma$ must be of the form $H=h \circ \pi_{p}$ for some $h \in C^{\infty}(G(p, n))$. It is clear that $\pi_{p}$ commutes with the left action of $M(n)$ on $G_{p, n} \times \mathbb{R}^{n}$ and $G(p, n)$, respectively. Similar considerations apply to the projection $\pi_{q}: G_{q, n} \times \mathbb{R}^{n} \rightarrow G(q, n)$.

Now $\mathfrak{m}(n)$ acts on $C^{\infty}\left(G_{p, n} \times \mathbb{R}^{n}\right)$ (and on $C^{\infty}\left(G_{q, n} \times \mathbb{R}^{n}\right)$ ) via vector fields: if $X \in M(n)$, and $H \in C^{\infty}\left(G_{p, n} \times \mathbb{R}^{n}\right)$, we set

$$
X \cdot H(\sigma, y)=\left.\frac{d}{d t} H(\exp (-t X) \cdot(\sigma, y))\right|_{t=0} .
$$


In particular, if $X \in \operatorname{so}(n)$, it follows that

$$
X \cdot H(\sigma, y)=X_{\sigma} H(\sigma, y)+X_{y} H(\sigma, y) .
$$

In the above, $X_{\sigma}$ and $X_{y}$ (which act on the first and second arguments, respectively) are the tangent vectors to the curves $\exp (-t X) \cdot \sigma$ in $G_{p, n}$ and $\exp (-t X) \cdot y$ in $\mathbb{R}^{n}$ at $t=0$. Similarly, if $v \in \mathbb{R}^{n}$, then

$$
\begin{aligned}
v \cdot H(\sigma, y) & =\left.\frac{d}{d t} H(\sigma, y-t v)\right|_{t=0} \\
& =v_{y} H(\sigma, y) .
\end{aligned}
$$

We extend the $\mathfrak{m}(n)$ action to an action of $\mathfrak{u}(\mathfrak{m}(n))$ on $C^{\infty}\left(G_{p, n} \times \mathbb{R}^{n}\right)$ and on $C^{\infty}\left(G_{q, n} \times \mathbb{R}^{n}\right)$.

Now assume that $f$ satisfies the Pfaffian equations $d \lambda\left(W_{I}\right) f=0$, and $d \lambda\left(V_{J}\right) f=$ 0 for all $I \in T_{2 m+2}, J \in T_{2 m+1}$. Let $F=f \circ \pi_{p}$. Since the $\mathfrak{u}(\mathfrak{m}(n))$-action commutes with $\pi_{p}$, we have $W_{I} \cdot F=0, V_{J} \cdot F=0$. Fix $I \in T_{2 m+2}$ and suppose $(\sigma, x) \in G_{p, n} \times \mathbb{R}^{n}$. Then

$$
\begin{aligned}
\left(\left(W_{I}\right)_{\sigma} F\right)(\sigma, x) & =\left(\left(W_{I}\right)_{\sigma} F\right)^{\tau(-x)}(\sigma, 0) \\
& =\left(\left(W_{I}\right)_{\sigma} F^{\tau(-x)}\right)(\sigma, 0),
\end{aligned}
$$

because $\tau(-x)$ acts on the second argument whereas $\left(W_{I}\right)_{\sigma}$ acts on the first. Now by (5.9) and (5.15),

$$
\begin{aligned}
\left(\left(W_{I}\right)_{\sigma} F^{\tau(-x)}\right)(\sigma, 0) & =\left(W_{I} \cdot F^{\tau(-x)}\right)(\sigma, 0) \\
& =\left(\left(\operatorname{Ad}(x) W_{I}\right) \cdot F\right)^{\tau(-x)}(\sigma, 0) \\
& =\left(\left(W_{I}-\sum_{P} e_{P I}(x) V_{P}\right) \cdot F\right)^{\tau(-x)}(\sigma, 0) \\
& =0 .
\end{aligned}
$$

Since we are presently assuming that $p+q>n$, we must have $\operatorname{rank}\left(G_{q, n}\right)=$ $n-q<\min (p, n-p)=\operatorname{rank}\left(G_{p, n}\right)$. Let $R_{q, p}$ denote the $O(n)$-equivariant (compact) Radon transform from $C^{\infty}\left(G_{q, n}\right)$ to $C^{\infty}\left(G_{p, n}\right)$ corresponding to the inclusion incidence relation. Then $R_{q, p}$ is injective and by the range conditions for $R_{q, p}$ (GK], Theorem 4.2 and subsequent remarks), there exists, for each $x \in \mathbb{R}^{n}$, a unique function $\Phi_{x}(\eta) \in C^{\infty}\left(G_{q, n}\right)$ for which

$$
F(\sigma, x)=\int_{\eta \supset \sigma} \Phi_{x}(\eta) d \eta
$$

We write $\Phi_{x}(\eta)$ as $\Phi(\eta, x)$ and the above as

$$
F(\sigma, x)=\int_{\eta \supset \sigma} \Phi(\eta, x) d \eta
$$

Next we prove that $\Phi \in C^{\infty}\left(G_{q, n} \times \mathbb{R}^{n}\right)$. For $q-p$ even, this follows from the inversion formula for $R_{q, p}$ given in [K]. Since there is no simple explicit inversion formula for $R_{q, p}$ when $p$ and $q$ do not have the same parity. In fact, in this case there exists an inversion formula given by Grinberg and Rubin $\mathrm{GrRu}$. However, their inversion formula contains differentiation in the sense of distributions, and therefore cannot be applied to prove the smoothness of the function $\Phi$. So we will show that $\Phi$ is smooth in general by diagonalizing $R_{q, p}$. (See $[\mathrm{K}]$ or $[\mathrm{Gr}]$ for details.) 
The spaces $L^{2}\left(G_{q, n}\right)$ and $L^{2}\left(G_{p, n}\right)$ decompose orthogonally into $O(n)$-invariant irreducible subspaces

$$
\begin{aligned}
& L^{2}\left(G_{q, n}\right)=\sum_{\delta \in \widehat{G}_{q, n}} \mathcal{H}_{\delta}, \\
& L^{2}\left(G_{p, n}\right)=\sum_{\delta^{\prime} \in \widehat{G}_{p, n}} \mathcal{H}_{\delta^{\prime}}^{\prime} .
\end{aligned}
$$

Here $\widehat{G}_{q, n}$ and $\widehat{G}_{p, n}$ are index sets corresponding to the equivalence classes of certain (spherical) representations of $O(n)$; we have $\widehat{G}_{q, n} \subset \widehat{G}_{p, n}$ ( $[\mathrm{Gr}$, [St]). Since $R_{q, p}$ is injective $(\underline{G r})$, one has $R_{q, p} \mathcal{H}_{\delta}=\mathcal{H}_{\delta}^{\prime}$ when $\delta \in \widehat{G}_{q, n}$; in fact, by Schur's lemma $R_{q, p}$ acts essentially as scalar multiplication on $\mathcal{H}_{\delta}$.

For each $\delta \in \widehat{G}_{q, n}$, let $d(\delta)=\operatorname{dim}\left(\mathcal{H}_{\delta}\right)$ and let $\left\{Y_{\delta \ell} \mid \ell=1, \ldots, d(\delta)\right\}$ be an orthonormal basis of $\mathcal{H}_{\delta}$. We can choose $Y_{\delta \ell}$ so that $\left|Y_{\delta \ell}(\eta)\right| \leq d(\delta)^{1 / 2}$ for all $\eta$, by the Peter-Weyl theorem. By the injectivity of $R_{q, p}$ there exists a constant $c_{\delta} \neq 0$ such that $Z_{\delta \ell}:=c_{\delta} R_{q, p} Y_{\delta \ell}, \ell=1, \ldots, d(\delta)$, is an orthonormal basis of $\mathcal{H}_{\delta}^{\prime}$. Let $\mu_{\delta}$ denote the highest weight of $\delta$, with respect to a choice of Weyl chamber in $G_{q, n}$. Then $c_{\delta}$ grows polynomially in $\mu_{\delta}$ in the sense that, for some constants $C_{0}$ and $M$, we have $\left|c_{\delta}\right| \leq C_{0}\left\|\mu_{\delta}\right\|^{M}$ for all $\delta,\|\|$ denoting the Killing form norm (G4]). We also note that $d(\delta)$ grows polynomially in $\mu_{\delta}$, by the Weyl dimension formula.

From (5.22) (and (5.201) ) we see that

$$
F(\sigma, x)=\sum_{\delta \in \widehat{G}_{q, n}} \sum_{\ell=1}^{d(\delta)} a_{\delta \ell}(x) Z_{\delta \ell}(\sigma)
$$

for certain coefficients $a_{\delta \ell}(x) \in C^{\infty}\left(\mathbb{R}^{n}\right)$. This series converges absolutely and uniformly on compact subsets of $G_{p, n} \times \mathbb{R}^{n}$ and can be differentiated term by term to any order in $x$ and $\sigma$. In addition, the $a_{\delta \ell}(x)$ are rapidly decreasing in the weight $\mu_{\delta}$ in the sense that for any compact set $K \subset \mathbb{R}^{n}$ and any positive integer $N$, there is a constant $C_{K, N}$ such that

$$
\left|a_{\delta \ell}(x)\right| \leq C_{K, N}\left\|\mu_{\delta}\right\|^{-N} \quad x \in K .
$$

The partial derivatives of $a_{\delta \ell}(x)$ (of any order) also satisfy a similar estimate.

By (5.20) and the estimates above we see that we can write $\Phi(\eta, x)$ as the absolutely convergent series

$$
\Phi(\eta, x)=\sum_{\delta \in \widehat{G}_{q, n}} \sum_{\ell=1}^{d(\delta)} c_{\delta} a_{\delta \ell}(x) Y_{\delta \ell}(\eta) .
$$

This series also converges uniformly on compact subsets of $G_{q, n} \times \mathbb{R}^{n}$. More precisely, since $c_{\delta}$ grows at most polynomially in $\delta$ (or more precisely in $\mu_{\delta}$ ), the coefficients $b_{\delta \ell}(x)=c_{\delta} a_{\delta \ell}(x)$ of $Y_{\delta \ell}(\eta)$ and their partial derivatives satisfy a decay estimate similar to (5.24). It also follows that $\Phi(\eta, x)$ is smooth in $(\eta, x)$, the smoothness in $\eta$ being a consequence of the rapid decrease of the coefficients $b_{\delta \ell}(x)$ in $\delta$.

We will next need to prove that the smooth function $\Phi$ satisfies the condition

$$
\Phi(\eta, x)=\Phi(\eta, x+v), \quad \text { for any } v \in \eta \text {. }
$$


This will imply the existence of a function $\varphi \in C^{\infty}(G(q, n))$ for which $\Phi=\varphi \circ \pi_{q}$. Assuming that such a function $\varphi$ exists, we have

$$
\Phi(\eta, x)=\Phi\left(\eta, P r_{\eta^{\perp}} x\right)=\varphi\left(\eta, P r_{\eta^{\perp}} x\right),
$$

for any $(\eta, x) \in G_{q, n} \times \mathbb{R}^{n}$, whence (5.20) can be written

$$
\begin{aligned}
f(\sigma, x) & =F(\sigma, x) \\
& =\int_{\eta \supset \sigma} \Phi(\eta, x) d \eta \\
& =\int_{\eta \supset \sigma} \Phi\left(\eta, \operatorname{Pr}_{\eta^{\perp}} x\right) d \eta \\
& =\int_{\eta \supset \sigma} \varphi\left(\eta, \operatorname{Pr}_{\eta^{\perp}} x\right) d \eta \\
& =\left(\mathcal{R}^{(q, p)} \varphi\right)(\sigma, x),
\end{aligned}
$$

for any $(\sigma, x) \in G(p, n)$, which of course shows that $f$ belongs to the range $\mathcal{R}^{(p, q)} C^{\infty}(G(q, n))$.

(5.26) will follow if we can show that for any two points $x \neq y$ in $\mathbb{R}^{n}, \Phi(\eta, x)=$ $\Phi(\eta, y)$ if $\eta$ is any element of $G_{q, n}$ containing $w=y-x$. Define the compact Grassmannians $G_{p}(w)=\left\{\sigma \in G_{p, n} \mid w \in \sigma\right\}$ and $G_{q}(w)=\left\{\eta \in G_{q, n} \mid w \in \eta\right\}$. Since $w \neq 0$, we have $G_{p}(w) \approx G_{p-1, n-1}$ and $G_{q}(w) \approx G_{q-1, n-1}$. We now claim that $\operatorname{rank}\left(G_{p}(w)\right) \geq \operatorname{rank}\left(G_{q}(w)\right)$. First, we have $2 q \geq n+1$ so $n-q \leq q-1$ and hence $\operatorname{rank}\left(G_{q}(w)\right)=\min (q-1, n-q)=n-q$. Moreover, we are presently assuming that $p+q \geq n+1$, so $n-q \leq p-1$, and of course, $n-q<n-p$. These imply that $\operatorname{rank}\left(G_{q}(w)\right)=n-q \leq \min (p-1, n-p)=\operatorname{rank}\left(G_{p}(w)\right)$, as claimed. Let $R_{q, p}(w): C^{\infty}\left(G_{q}(w)\right) \rightarrow C^{\infty}\left(G_{p}(w)\right)$ be the naturally defined integral transform arising from the inclusion relation. From the rank data, we see that this transform is injective.

Define smooth functions $\psi_{x}$ and $\psi_{y}$ on $G_{q}(w)$ by setting $\psi_{x}(\eta)=\Phi(\eta, x)$ and $\psi_{y}(\eta)=\Phi(\eta, y)$, for any $\eta \in G_{q}(w)$. Let $\sigma \in G_{p}(w)$. We have

$$
\begin{aligned}
F(\sigma, x) & =\int_{\eta \supset \sigma} \Phi(\eta, x) d \eta \\
& =\int_{\eta \supset \sigma} \psi_{x}(\eta) d \eta \\
& =\left(R_{q, p}(w) \psi_{x}\right)(\sigma),
\end{aligned}
$$

and also

$$
F(\sigma, y)=\left(R_{q, p}(w) \psi_{y}\right)(\sigma) .
$$

Since $F(\sigma, x)=F(\sigma, y)$ for all $\sigma \in G_{p}(w)$, we have $R_{q, p}(w) \psi_{x}=R_{q, p}(w) \psi_{y}$ and hence (by the injectivity of $\left.R_{q, p}\right) \psi_{x}=\psi_{y}$. This shows that $\Phi(\eta, x)=\Phi(\eta, y)$ for all $\eta \in G_{q}(w)$ and completes the proof of (5.26).

Remarks. 1. The proof of Theorem 5.1 (unfortunately) breaks down in the case $p+q=n$ (still assuming, of course, that $\operatorname{rank}(G(q, n))<\operatorname{rank}(G(p, n)))$, because in this case $\operatorname{rank}\left(G_{q}(w)\right)=\min (q-1, n-q)=n-q=p>p-1 \geq \operatorname{rank}\left(G_{p}(w)\right)$. Hence $R_{q, p}(w)$ is no longer injective.

Theorem 6.1 below suggests, however, that when $p+q=n$ and $\operatorname{rank}(G(q, n))$ $<\operatorname{rank}(G(p, n))$, a stronger set of range conditions apply. Specifically, it is likely 
that $\mathcal{R}^{(q, p)} C^{\infty}(G(q, n))$ consists of those functions $f \in C^{\infty}(G(p, n))$ annihilated by the operators $d \lambda\left(V_{J}\right), J \in T_{2 m+1}$, or, equivalently, by the single $M(n)$-invariant differential operator $d \lambda\left(\sum_{J \in T_{2 m+1}} V_{J}^{2}\right)$, of order $2 m+2$. We will provide a proof of this assertion in the special case $p=1, q=n-1$.

2. In Theorem [5.1, the order $m+1$ Pfaffian equations $d \lambda\left(W_{I}\right) f=0$ and $d \lambda\left(V_{J}\right) f=0$ are both essential in characterizing the range of $\mathcal{R}^{(p, q)}$. (See Remark 3 below.) This is in contrast with the likely range characterization for $\mathcal{R}^{(q, p)}$ in the case $p+q=n$, given in the preceding remark, as well as the range characterization in the case of the transform $\mathcal{R}^{(p, q)}={ }^{t} \mathcal{R}^{(q, p)}: \mathcal{S}(G(p, n)) \rightarrow \mathcal{S}(G(q, n))$ when $\operatorname{rank}(G(p, n))<\operatorname{rank}(G(q, n))$, which was given in [GK], Theorem 7.7, and summarized below.

Let $\mathcal{S}(G(p, n))$ and $\mathcal{S}(G(q, n))$ denote the Schwartz spaces on $G(p, n)$ and $G(q, n)$, respectively ( $[\mathrm{Ri}])$. Let $r$ denote the rank of $G(p, n)$. According to the above-quoted theorem, if $r=\operatorname{rank}(G(p, n))<\operatorname{rank}(G(q, n))$, then the range $\mathcal{R}^{(p, q)} \mathcal{S}(G(p, n))$ consists precisely of those functions $\varphi \in \mathcal{S}(G(q, n))$ for which $d \nu\left(V_{J}\right) \varphi=0$ for all $J \in T_{2 r+1}$; alternatively, $\mathcal{R}^{(p, q)} \mathcal{S}(G(p, n))$ is given by the set of all $\varphi \in \mathcal{S}(G(q, n))$ annihilated by the $M(n)$-invariant differential operator $d \nu\left(\sum_{J \in T_{2 r+1}} V_{J}^{2}\right)$. While the functions in the range of $\mathcal{R}^{(p, q)}$ also satisfy the equations $d \nu\left(W_{I}\right) \varphi=0$ for $I \in T_{2 r+2}$, these equations are not needed in the range characterization.

3. The following example shows why the equations $d \lambda\left(W_{I}\right) f=0$ are needed in the range characterization of $\mathcal{R}^{(q, p)}$ in Theorem 5.1. Let us assume the hypotheses of Theorem 5.1 namely that $p<q, p+q>n$, and $\operatorname{rank}(G(p, n)>\operatorname{rank}(G(q, n)$. The relation $q>\left[\frac{n}{2}\right]$ implies that $2 q>n$, so $q>n-q$, and thus $\operatorname{rank}\left(G_{q, n}\right)=$ $n-q<\min (p, n-p)=\operatorname{rank}\left(G_{p, n}\right)$.

Consider the orthogonal decompositions (15.21) and (5.22) of $L^{2}\left(G_{q, n}\right)$ and $L^{2}\left(G_{p, n}\right)$, respectively. As mentioned earlier, we have $R_{q, p} \mathcal{H}_{\delta}=\mathcal{H}_{\delta}^{\prime}$ when $\delta \in \widehat{G}_{q, n}$. Now the rank data imply that $\widehat{G}_{q, n} \varsubsetneqq \widehat{G}_{p, n}$. Fix any $\delta_{0} \in \widehat{G}_{p, n} \backslash \widehat{G}_{q, n}$ and any $\psi \neq 0, \in \mathcal{H}_{\delta_{0}}^{\prime}$. Then $\psi \notin R_{q, p}\left(C^{\infty}\left(G_{q, n}\right)\right)$. Define the function $h$ on $G(p, n)$ by setting $h(\sigma, x)=\psi(\sigma)$ for any $\sigma \in G_{p, n}$ and $x \in \sigma^{\perp}$. Then $h$ is smooth on $G(p, n)$ and invariant under the translations $\tau(v), v \in \mathbb{R}^{n}$. As a result, we clearly have $d \lambda\left(V_{J}\right) h=0$ for all $J \in T_{2 m+1}$. On the other hand, there is no function $\varphi \in C^{\infty}(G(q, n))$ for which $h(\sigma, 0)=\int_{\eta \supset \sigma, \eta \in G_{q, n}} \varphi(\eta, 0) d \eta$; in particular, $h \notin \mathcal{R}^{(q, p)} C^{\infty}(G(q, n))$.

\section{The SPECIAL CASE $p+q=n$}

Let us now focus our attention on the case $1 \leq p<q<n$, with $p+q=n$. Then $\operatorname{rank}(G(p, n))=p+1>n-q=\operatorname{rank}(G(q, n))$. Suppose now that $f \in C^{\infty}(G(p, n))$ satisfies the Pfaffian system $d \lambda\left(V_{J}\right) f=0$ for all $J \in T_{2 m+1}$. As usual, we have set $m=\operatorname{rank}(G(q, n)) \quad(=p$ in the present case $)$. Consider the pull-back $F=f \circ \pi_{p}$. This function is of course smooth on $G_{p, n} \times \mathbb{R}^{n}$. Now $\operatorname{since} \operatorname{rank}\left(G_{p, n}\right)=\operatorname{rank}\left(G_{q, n}\right)$ here, the transform $R_{q, p}: C^{\infty}\left(G_{q, n}\right) \rightarrow C^{\infty}\left(G_{p, n}\right)$ is a bijection ([Gr] $)$. Thus for each $x \in \mathbb{R}^{n}$, there exists a function $\Phi_{x} \in C^{\infty}\left(G_{q, n}\right)$ such that

$$
F(\sigma, x)=\int_{\eta \supset \sigma} \Phi_{x}(\eta) d \eta
$$


Writing $\Phi_{x}(\eta)=\Phi(\eta, x)$, we may rewrite the above as

$$
F(\sigma, x)=\int_{\eta \supset \sigma} \Phi(\eta, x) d \eta .
$$

As in the proof of Theorem 5.1 it is easy to see (using either the inversion formula for $R_{p, q}$, or differentiating inside the integral sign) that $\Phi \in C^{\infty}\left(G_{q, n} \times \mathbb{R}^{n}\right)$.

Our objective, as with equation (5.26), is to show that

$$
\Phi(\eta, x)=\Phi(\eta, x+v)
$$

for any $v \in \eta$. Of course, we can no longer apply the argument in equation (5.28) because $\operatorname{rank}\left(G_{q}(w)\right)>\operatorname{rank}\left(G_{p}(w)\right)$ by Remark 1 following Theorem 5.1 Nonetheless, condition (6.3) for $\Phi$ should allow us to conclude (as in equations (5.26) and (5.27) for the case $p+q>n$ ) that $\Phi=\varphi \circ \pi_{q}$ for some $\varphi \in C^{\infty}\left(G_{q, n}\right)$, so that $\mathcal{R}^{(q, p)} \varphi=f$. Now let $\nabla_{x} \Phi(\eta, x)$ denote the gradient of $\Phi$ with respect to its second argument: $\nabla_{x} \Phi(\eta, x)=\left(\left(E_{1} \cdot \Phi\right)(\eta, x), \cdots,\left(E_{n} \cdot \Phi\right)(\eta, x)\right) \in \mathbb{R}^{n}$. Then (6.3) is equivalent to the condition $\nabla_{x} \Phi(\eta, x) \perp \eta$.

We proceed to obtain some properties of $\Phi$ from the Pfaffian equations $d \lambda\left(V_{J}\right) f=$ 0 . These conditions will allow us to derive the equation (6.3) in the case $p=1, q=$ $n-1$, thereby proving the range theorem, at least in this special case. Now the projection $\pi_{p}$ commutes with the left action of $M(n)$ on the space $G(p, n)$ and $G_{p, n} \times \mathbb{R}^{n}$, so we obtain

$$
V_{J} \cdot F=0,
$$

for each $J \in T_{2 m+1}$. We provide a more explicit expression for (6.4) as follows. Let $(\sigma, x) \in G_{p, n} \times \mathbb{R}^{n}$ and $J: j_{1}<j_{2}<\cdots<j_{2 m+1}$ in $T_{2 m+1}=T_{2 p+1}$,

$$
\begin{aligned}
0 & =\left(V_{J} \cdot F\right)(\sigma, x) \\
& =\left(\left(\operatorname{Ad}(-x) V_{J}\right) F^{\tau(-x)}\right)(\sigma, 0) \\
& =\left(V_{J} F^{\tau(-x)}\right)(\sigma, 0) \\
& =\sum_{r=1}^{2 p+1}(-1)^{r-1}\left(\left(W_{J \backslash\left\{j_{r}\right\}} E_{j_{r}}\right) F^{\tau(-x)}\right)(\sigma, 0) \\
& =\sum_{r=1}^{2 p+1}(-1)^{r-1}\left(\left(\left(W_{J \backslash\left\{j_{r}\right\}}\right)_{\sigma} \circ E_{j_{r}}\right) F^{\tau(-x)}\right)(\sigma, 0) \\
& =\sum_{r=1}^{2 p+1}(-1)^{r-1}\left(E_{j_{r}} \cdot\left(\left(W_{J \backslash\left\{j_{r}\right\}}\right)_{\sigma} F\right)^{\tau(-x)}\right)(\sigma, 0) \\
& =\sum_{r=1}^{2 p+1}(-1)^{r-1}\left(\left(E_{j_{r}}\right)_{x} \circ\left(W_{J \backslash\left\{j_{r}\right\}}\right)_{\sigma} F\right)(\sigma, x) .
\end{aligned}
$$

Apply (6.5) to (6.2) and differentiate inside the integral sign, noting that $R_{q, p}$ is $O(n)$-equivariant. This gives

$$
0=\int_{\eta \supset \sigma} \sum_{r=1}^{2 p+1}(-1)^{r-1}\left(\left(E_{j_{r}}\right)_{x} \circ\left(W_{J \backslash\left\{j_{r}\right\}}\right)_{\eta} \Phi\right)(\eta, x) d \eta,
$$


for each $(\sigma, x) \in G_{p, n} \times \mathbb{R}^{n}$. $R_{q, p}$ being injective, we conclude that the expression inside the integral sign vanishes; i.e.,

$$
\sum_{r=1}^{2 p+1}(-1)^{r-1}\left(\left(E_{j_{r}}\right)_{x} \circ\left(W_{J \backslash\left\{j_{r}\right\}}\right)_{\eta} \Phi\right)(\eta, x)=0
$$

for each $(\eta, x) \in G_{q, n} \times \mathbb{R}^{n}$ and each $J \in T_{2 p+1}$.

Now the condition $\nabla_{x} F(\sigma, x) \perp \sigma$ (which is equivalent to $F(\sigma, x)=F(\sigma, x+w)$ for all $w \in \sigma$ ) and equation (6.2) leads us to the following integral condition on $\Phi$ :

$$
0=\int_{\eta \supset \sigma}\left\langle\nabla_{x} \Phi(\eta, x), w\right\rangle d \eta
$$

for each $w \in \sigma$. Here $\langle\cdot, \cdot\rangle$ denotes the usual inner product on $\mathbb{R}^{n}$.

Let us now fix $x \in \mathbb{R}^{n}$ and consider the section $\widetilde{\Phi}$ on the trivial bundle $\pi$ : $G_{q, n} \times \mathbb{R}^{n} \rightarrow G_{q, n}$ given by $\widetilde{\Phi}(\eta)=\nabla_{x} \Phi(\eta, x)$. Write $\widetilde{\Phi}(\eta)=\left(\widetilde{\Phi}_{1}(\eta), \cdots, \widetilde{\Phi}_{n}(\eta)\right)$. Equations (6.7) and (6.8) imply that $\widetilde{\Phi}$ satisfies the two conditions

$$
\begin{aligned}
& \sum_{r=1}^{2 p+1}(-1)^{r-1}\left(W_{J \backslash\left\{j_{r}\right\}} \widetilde{\Phi}_{j_{r}}\right)(\eta)=0, \\
& \int_{\eta \supset \sigma}\langle\widetilde{\Phi}(\eta), w\rangle d \eta=0,
\end{aligned}
$$

for each $w \in \sigma$. Our objective is to prove that $\widetilde{\Phi}(\eta) \perp \eta$, given these two conditions.

It is more convenient to orthogonally decompose $\widetilde{\Phi}(\eta)$ into components parallel and perpendicular to $\eta$ : write

$$
\widetilde{\Phi}(\eta)=\bar{\Phi}(\eta)+\Psi(\eta)
$$

where $\bar{\Phi}(\eta) \perp \eta$ and $\Psi(\eta) \in \eta$. We of course wish to prove that $\Psi(\eta)=0$ for each $\eta \in G_{q, n}$.

The following technical lemma allows us to reduce the problem to one involving the section $\Psi$ :

Lemma 6.1. Let $J \in T_{2 m+1} ; J: j_{1}<j_{2}<\cdots<j_{2 m+1}$. For any $\eta \in G_{q, n}$, we have

$$
\sum_{r=1}^{2 m+1}(-1)^{r-1}\left(W_{J \backslash\left\{j_{r}\right\}} \bar{\Phi}_{j_{r}}\right)(\eta)=0 .
$$

Proof. By hypothesis, $\bar{\Phi}(\eta) \perp \eta$. Let $\tau: G_{p, n} \rightarrow G_{q, n}, \tau(\sigma)=\sigma^{\perp}$ be the natural bijection of $G_{p, n}$ onto $G_{q, n}$. Then the mapping $T=\bar{\Phi} \circ \tau$ is a smooth section of the universal bundle $U_{p, n}=\left\{(\sigma, x) \in G_{p, n} \times \mathbb{R}^{n} \mid x \in \sigma\right\}$ of $G_{p, n}$. We identify $\mathbb{R}^{n}$ with the space of $n \times 1$ column vectors and we write $T(\sigma)=\left(T_{1}(\sigma), \cdots, T_{n}(\sigma)\right)$. Since $\tau$ is $S O(n)$-equivariant, (6.11) is equivalent to the condition

$$
\sum_{r=1}^{2 p+1}(-1)^{r-1}\left(W_{J \backslash\left\{j_{r}\right\}} T_{j_{r}}\right)(\sigma)=0,
$$

for any $\sigma \in G_{p, n}$, and any $J \in T_{2 p+1}$.

In order to prove (6.12), we first note that $S O(n)$ acts on smooth sections of the universal bundle $U_{p, n} \rightarrow G_{p, n}$ via $(u \cdot F)(\sigma)=(u F)\left(u^{-1} \cdot \sigma\right)$, for any $u \in$ $S O(n), \sigma \in G_{p, n}$. 
Now for any $J \in T_{2 p+1}$, we denote the left-hand side of (6.12) by $T_{J}$. (Note that this is a slight abuse of notation.)

Claim. $(u \cdot T)_{J}(\sigma)=\sum_{M \in T_{2 p+1}} \operatorname{det}\left(u_{J M}\right) T_{M}\left(u^{-1} \cdot \sigma\right)$, for any $u \in S O(n), \sigma \in G_{p, n}$.

We prove this claim by a straightforward, but somewhat lengthy, calculation, as follows:

$$
\begin{aligned}
(u \cdot T)_{J}(\sigma) & =\sum_{r=1}^{2 p+1}(-1)^{r-1} W_{J \backslash\left\{j_{r}\right\}}(u \cdot T)_{j_{r}}(\sigma) \\
& =\sum_{r=1}^{2 p+1}(-1)^{r-1}\left(W_{J \backslash\left\{j_{r}\right\}}\right)_{\sigma}\left(\sum_{l=1}^{n} u_{j_{r}, l} T_{l}\left(u^{-1} \cdot \sigma\right)\right) \\
& =\sum_{r=1}^{2 p+1} \sum_{l=1}^{n}(-1)^{r-1} u_{j_{r}, l}\left(W_{J \backslash\left\{j_{r}\right\}}\right)_{\sigma} T_{l}\left(u^{-1} \cdot \sigma\right) \\
& =\sum_{r=1}^{2 p+1} \sum_{l=1}^{n}(-1)^{r-1} u_{j_{r}, l}\left(\left(\operatorname{Ad}\left(u^{-1}\right) W_{J \backslash\left\{j_{r}\right\}}\right) T_{l}\right)\left(u^{-1} \cdot \sigma\right) \\
& =\sum_{r=1}^{2 p+1} \sum_{l=1}^{n} \sum_{L \in T_{2 p}}(-1)^{r-1} u_{j_{r}, l} \operatorname{det}\left(u^{-1}\right)_{L, J \backslash\left\{j_{r}\right\}}\left(W_{L} T_{l}\right)\left(u^{-1} \cdot \sigma\right) \\
& =\sum_{l=1}^{n} \sum_{L \in T_{2 p}} \sum_{r=1}^{2 p+1}(-1)^{r-1} u_{j_{r}, l} \operatorname{det}(u)_{J \backslash\left\{j_{r}\right\}, L}\left(W_{L} T_{l}\right)\left(u^{-1} \cdot \sigma\right) .
\end{aligned}
$$

Let $L \in T_{2 p}$ be given by $L: l_{1}<l_{2}<\cdots<l_{2 p}$. Then

$$
\sum_{r=1}^{2 p+1}(-1)^{r-1} u_{j_{r}, l} \operatorname{det}(u)_{J \backslash\left\{j_{r}\right\}, L}=\left|\begin{array}{cccc}
u_{j_{1}, l} & u_{j_{1}, l_{1}} & \cdots & u_{j_{1}, l_{2 p}} \\
u_{j_{2}, l} & u_{j_{2}, l_{1}} & \ldots & u_{j_{2}, l_{2 p}} \\
& \cdots & \cdots & \\
u_{j_{2 p+1}, l} & u_{j_{2 p+1}, l_{1}} & \cdots & u_{j_{2 p+1}, l_{2 p}}
\end{array}\right| .
$$

The latter determinant equals zero if $l \in L$, and equals $\epsilon(l, L) \operatorname{det}\left(u_{J, L \cup\{l\}}\right)$ if $l \notin L$. Thus the last expression in (6.13) equals

$$
\sum_{\substack{1 \leq l \leq n \\ L \in T_{2 p}, l \notin L}} \epsilon(l, L) \operatorname{det}\left(u_{J, L \cup\{l\}}\right)\left(W_{L} T_{l}\right)\left(u^{-1} \cdot \sigma\right) .
$$

We can rewrite the above sum by setting $M=L \cup\{l\}$ (for $l \notin L$, so $M \in T_{2 p+1}$ ), to obtain

$$
\begin{aligned}
& \sum_{M \in T_{2 p+1}} \operatorname{det}\left(u_{J M}\right) \sum_{k=1}^{2 p+1}(-1)^{k-1}\left(W_{M \backslash\left\{m_{k}\right\}} T_{m_{k}}\right)\left(u^{-1} \cdot \sigma\right) \\
= & \sum_{M \in T_{2 p+1}} \operatorname{det}\left(u_{J M}\right)\left(T_{M}\right)\left(u^{-1} \cdot \sigma\right) .
\end{aligned}
$$

In the above, we have written $M: m_{1}<m_{2}<\cdots<m_{2 p+1}$. This completes the proof of the claim.

The condition (6.12), which is all that we need in order to prove Lemma 6.1, is equivalent to the condition $T_{J}(\sigma)=0$ for each $J \in T_{2 p+1}, \sigma \in G_{p, n}$. So fix $J$ and 
$\sigma$. Let $\sigma_{0}=\mathbb{R} e_{1} \oplus \cdots \oplus \mathbb{R} e_{p} \in G_{p, n}$, and choose an element $u \in S O(n)$ such that $u^{-1} \cdot \sigma_{0}=\sigma$. Then by the claim above

$$
\begin{aligned}
T_{J}(\sigma) & =\left(u \cdot\left(u^{-1} \cdot T\right)\right)_{J}(\sigma) \\
& =\sum_{M \in T_{2 p+1}} \operatorname{det}\left(u_{J M}\right)\left(u^{-1} \cdot T\right)_{M}\left(\sigma_{0}\right) .
\end{aligned}
$$

Let $S=u^{-1} \cdot T ; S$ is a section of the universal bundle $U_{p, n} \rightarrow G_{p, n}$. It suffices for us to prove that $S_{M}\left(\sigma_{0}\right)=0$ for each $M \in T_{2 p+1}$.

Again writing $M: m_{1}<m_{2}<\cdots<m_{2 p+1}$, we have

$$
S_{M}\left(\sigma_{0}\right)=\sum_{k=1}^{2 p+1}(-1)^{k-1}\left(W_{M \backslash\left\{m_{k}\right\}} S_{m_{k}}\right)\left(\sigma_{0}\right) .
$$

We show that each summand in the right-hand side above equals zero. This simply involves an index count. Now $\left(W_{M \backslash\left\{m_{k}\right\}} S_{m_{k}}\right)\left(\sigma_{0}\right)$ is a sum of terms of the form

$$
\left(X_{r_{1}, r_{2}} \cdots X_{r_{2 p-1}, r_{2 p}} S_{m_{k}}\right)\left(\sigma_{0}\right)
$$

where $\left(r_{1}, \cdots, r_{2 p}\right) \in \mathfrak{S}\left(M \backslash\left\{m_{k}\right\}\right)$. If $m_{k}>p$, then we write (6.20)

$$
\begin{aligned}
& \left(X_{r_{1}, r_{2}} \cdots X_{r_{2 p-1}, r_{2 p}} S_{m_{k}}\right)\left(\sigma_{0}\right) \\
& =\left\{\frac{\partial^{p}}{\partial t_{1} \cdots \partial t_{p}} S_{m_{k}}\left(\exp \left(-t_{1} X_{r_{1}, r_{2}}\right) \cdots \exp \left(-t_{p} X_{r_{2 p-1}, r_{2 p}}\right) \cdot \sigma_{0}\right)\right\}_{t_{1}=\cdots=t_{p}=0} .
\end{aligned}
$$

In this case, $\exp \left(-t_{1} X_{r_{1}, r_{2}}\right) \cdots \exp \left(-t_{p} X_{r_{2 p-1}, r_{2 p}}\right) \cdot \sigma_{0}$ is a $p$-plane in $e_{m_{k}}^{\perp}$, so

$$
S_{m_{k}}\left(\exp \left(-t_{1} X_{r_{1}, r_{2}}\right) \cdots \exp \left(-t_{p} X_{r_{2 p-1}, r_{2 p}}\right) \cdot \sigma_{0}\right)=0 .
$$

If $m_{k} \leq p$, it is easy to see that there exists a pair $\left(r_{2 k-1}, r_{2 k}\right)$ of indices belonging to $\{p+1, \cdots, n\}$. Then in this case we write

$$
\begin{aligned}
& \left(X_{r_{1}, r_{2}} \cdots X_{r_{2 p-1}, r_{2 p}} S_{m_{k}}\right)\left(\sigma_{0}\right) \\
& =\left.\frac{d}{d t}\left(X_{r_{1}, r_{2}} \cdots \widehat{X}_{r_{2 k-1}, r_{2 k}} \cdots X_{r_{2 p-1}, r_{2 p}} S_{m_{k}}\right)\left(\exp \left(-t X_{r_{2 k-1}, r_{2 k}}\right) \cdot \sigma_{0}\right)\right|_{t=0} .
\end{aligned}
$$

But $\exp \left(-t X_{r_{2 k-1}, r_{2 k}}\right) \cdot \sigma_{0}=\sigma_{0}$, so the above term also equals zero. This completes the proof of Lemma 6.1

Let us write $\Psi(\eta)=\left(\Psi_{1}(\eta), \cdots, \Psi_{n}(\eta)\right)$. $\Psi$ is of course a smooth section of the universal bundle $U_{q, n} \rightarrow G_{q, n}$; by (6.9) and (6.11), $\Psi$ also satisfies the Pfaffian system

$$
\sum_{r=1}^{2 p+1}(-1)^{r-1}\left(W_{J \backslash\left\{j_{r}\right\}} \Psi_{j_{r}}\right)(\eta)=0,
$$

for each $\eta \in G_{q, n}$ and $J \in T_{2 p+1}$. In the notation introduced before the claim during the proof of Lemma 6.1, $\Psi$ thus satisfies the system $\Psi_{J}=0$ for each $J \in T_{2 p+1}$. In addition, the section $\bar{\Phi}$ given in (6.10) trivially satisfies the integral condition

$$
\int_{\eta \supset \sigma}\langle\bar{\Phi}(\eta), w\rangle d \eta=0 \quad\left(\sigma \in G_{p, n}\right)
$$


for each $w \in \sigma$, since $\bar{\Phi}(\eta) \perp \eta$, so by (6.9), we must have

$$
\int_{\eta \supset \sigma}\langle\Psi(\eta), w\rangle d \eta=0 \quad\left(\sigma \in G_{p, n}\right)
$$

for all $w \in \sigma$.

The proof of the range theorem for $\mathcal{R}^{(p, q)}$, where $p+q=n$, thus reduces to showing that any smooth section $\Psi$ of the universal bundle $U_{q, n} \rightarrow G_{q, n}$ satisfying

$$
\Psi_{J}=0 \quad \text { for all } J \in T_{2 p+1}
$$

and

$$
\int_{\eta \supset \sigma}\langle\Psi(\eta), w\rangle d \eta=0 \quad\left(\sigma \in G_{p, n}, w \in \sigma\right)
$$

must vanish.

We prove this last statement in the case $q=n-1$. Assume then that $\Psi$ is a section of the universal bundle $U_{n-1, n} \rightarrow G_{n-1, n}$ satisfying $\Psi_{J}=0$ for all $J \in T_{3}$ and

$$
\int_{\eta \supset \sigma}\langle\Psi(\eta), v\rangle d \eta=0 \quad\left(\sigma \in G_{1, n}, v \in \sigma\right) .
$$

We wish to show that $\Psi$ vanishes.

We identify $G_{n-1, n}$ with $\mathbb{R P}^{n-1}$. Let $\widetilde{\pi}$ denote the (twofold) projection of $\mathbf{S}^{n-1}$ onto $G_{n-1, n}$ given by $\widetilde{\pi}(\omega)=\omega^{\perp}$, and let $T=\Psi \circ \widetilde{\pi}$. Since $T(\omega) \perp \omega$ and $T(-\omega)=T(\omega)$ for all $\omega \in \mathbf{S}^{n-1}$, we may consider $T$ to be a smooth, even vector field on $\mathbf{S}^{n-1}$. Since $\tilde{\pi}$ commutes with the $S O(n)$-actions on $\mathbf{S}^{n-1}$ and $G_{n-1, n}$, respectively, the conditions (6.26) and (6.27) on $\Psi$ imply that

$$
\begin{gathered}
T_{J}=0, \\
\int_{\omega \in \mathbf{S}^{n-1}, \omega \perp v}\langle T(\omega), v\rangle d_{v} \omega=0,
\end{gathered}
$$

for each $v \in \mathbf{S}^{n-1}$. Here $d_{v} \omega$ denotes the area element on the totally geodesic submanifold $A(v)=v^{\perp} \cap \mathbf{S}^{n-1}$. Writing $T(\omega)=\left(T_{1}(\omega), \cdots, T_{n}(\omega)\right)$, the condition (6.29) becomes

$$
\left(X_{i j} T_{k}-X_{i k} T_{j}+X_{j k} T_{i}\right)(\omega)=0,
$$

for all $1 \leq i<j<k \leq n$.

First we show that these equations imply that $T=\operatorname{grad} f$ for a smooth function $f$ on $\mathbf{S}^{n-1}$. For this, we extend $T$ to a smooth vector field $\widetilde{T}$ on a small spherical shell $1-\epsilon<\|x\|<1+\epsilon$ in $\mathbb{R}^{n}$, with $\widetilde{T}(r \omega)=T(\omega)$ for all $r \in(1-\epsilon, 1+\epsilon)$. Since $\widetilde{T}$ is radially constant, equation (6.31) still holds on the spherical shell, with $\widetilde{T}$ replacing $T$ and $x$ replacing $\omega$. From the natural identification of vector fields and 1-forms on $\mathbb{R}^{n}, \widetilde{T}$ corresponds to the 1 -form $\widetilde{\tau}=\sum_{i=1}^{n} T_{i} d x_{i}$, and $T$ corresponds to the pull-back $\tau$ of $\widetilde{\tau}$ to $\mathbf{S}^{n-1}$. Now $d \widetilde{\tau}=\sum_{i<j}\left(\frac{\partial \bar{T}_{j}}{d x_{i}}-\frac{\partial T_{i}}{d x_{j}}\right) d x_{i} d x_{j} ; d \tau$ has the same expression. We show that $d \tau=0$ by showing that $d \tau$ vanishes on each set of a finite open cover of $\mathbf{S}^{n-1}$. On the open subset $x_{n} \neq 0$ of $\mathbf{S}^{n-1}$, we can replace 
$d x_{n}$ by $-\frac{1}{x_{n}} \sum_{k=1}^{n-1} x_{k} d x_{k}$; the coefficient of $d x_{i} d x_{j}$ then becomes

$$
\begin{aligned}
& \left(\frac{\partial T_{j}}{d x_{i}}-\frac{\partial T_{i}}{d x_{j}}\right)-\frac{x_{j}}{x_{n}}\left(\frac{\partial T_{n}}{d x_{i}}-\frac{\partial T_{i}}{d x_{n}}\right)+\frac{x_{i}}{x_{n}}\left(\frac{\partial T_{n}}{d x_{j}}-\frac{\partial T_{j}}{d x_{n}}\right) \\
& =\frac{1}{x_{n}}\left[\left(x_{i} \frac{\partial T_{n}}{d x_{j}}-x_{j} \frac{\partial T_{n}}{d x_{i}}\right)-\left(x_{i} \frac{\partial T_{j}}{d x_{n}}-x_{n} \frac{\partial T_{j}}{d x_{i}}\right)+\left(x_{j} \frac{\partial T_{i}}{d x_{n}}-x_{n} \frac{\partial T_{i}}{d x_{j}}\right)\right] \\
& =\frac{1}{x_{n}}\left[X_{i j} \cdot T_{n}-X_{i n} \cdot T_{j}+X_{j n} \cdot T_{i}\right] \\
& =0, \quad \text { (by (6.31) }) .
\end{aligned}
$$

We can likewise show that $d \tau$ vanishes on each open subset $x_{i} \neq 0$ of $\mathbf{S}^{n-1}$; hence $d \tau=0$ on $\mathbf{S}^{n-1}$. Since the first cohomology group of $\mathbf{S}^{n-1}$ vanishes, this implies that $\tau=d f$ for some smooth function $f$ on $\mathbf{S}^{n-1}$; under the identification of $T$ with $\tau$, we get $T=\operatorname{grad} f$. Resolving $f$ into odd and even components, we note that the even component must be constant, so we can assume that $f$ is an odd function on $\mathbf{S}^{n-1}$.

For each $v \in \mathbf{S}^{n-1}$, let $H(v)$ denote the hemisphere opposite to $v: H(v)=\{\omega \in$ $\left.\mathbf{S}^{n-1} \mid\langle\omega, v\rangle<0\right\}$. Since $v$ is the outward-pointing unit normal on its boundary in $\mathbf{S}^{n-1}$, we can apply the divergence theorem (on $\mathbf{S}^{n-1}$ ) to the integral in (6.30) to obtain

$$
0=\int_{A(v)}\langle v, \operatorname{grad} f(\omega)\rangle d_{v} \omega=\int_{H(v)} L_{\mathbf{S}^{n-1}} f(\omega) d \omega .
$$

Here $L_{\mathbf{S}^{n-1}}$ denotes the Laplace-Beltrami operator and $d \omega$ denotes the volume element on $\mathbf{S}^{n-1}$. (6.33) shows that $L_{\mathbf{S}^{n-1}} f$ is a smooth odd function on $\mathbf{S}^{n-1}$ whose integral vanishes on all hemispheres. The following lemma shows that the only such function is zero. Since $\mathbf{S}^{n-1}$ is compact, this will imply that $f$ is constant, hence zero, so that $T=\operatorname{grad}_{\mathbf{S}^{n-1}} f=0$.

Lemma 6.2. Let $\gamma$ denote the hemisphere transform on $\mathbf{S}^{n-1}$,

$$
(\gamma(h))(v)=\int_{H(v)} h(\omega) d \omega, \quad \text { for } h \in C^{\infty}\left(\mathbf{S}^{n-1}\right) .
$$

Then $\gamma(h)=0$ only if $h$ is even.

Proof. Since $\gamma$ commutes with the action of $S O(n)$ on $\mathbf{S}^{n-1}$, it can be diagonalized. Let $\mathcal{H}_{j}$ denote the space of degree $j$ spherical harmonics on $\mathbf{S}^{n-1}$, for $j=0,1,2, \cdots$. $S O(n)$ acts irreducibly on each $\mathcal{H}_{j}$ so $\gamma$ must equal a scalar operator $c_{j}$ there. $c_{j}$ can be calculated by integrating a zonal harmonic in $\mathcal{H}_{j}$ over an appropriate hemisphere; this gives

$$
c_{j}=\int_{0}^{1} C_{j}^{\frac{n-2}{2}}(x)\left(1-x^{2}\right)^{\frac{n-3}{2}} d x
$$

where $C_{j}^{\nu}$ is a Gegenbauer polynomial of degree $j$. From Formula 7.311, page 826 of the book by Gradshteyn and Ryzhik GR, we obtain

$$
c_{j}= \begin{cases}\Gamma\left(\frac{n-1}{2}\right) & j=0, \\ 0 & j \text { even, } \\ \frac{\Gamma(n-2+j) \Gamma\left(\frac{n-1}{2}\right)}{j ! \Gamma(n-2) \Gamma\left(-\frac{j}{2}\right) \Gamma\left(\frac{n+j}{2}\right)} & j \text { odd. }\end{cases}
$$

This establishes the lemma. 
Here we note that more information about the hemispherical transform can be found in [Ru1].

From the argument above, we obtain the following range characterization theorem for $\mathcal{R}^{(n-1,1)}$.

Theorem 6.1. The image $\mathcal{R}^{(n-1,1)}\left(C^{\infty}(G(n-1, n))\right)$ equals the space $\{f \in$ $C^{\infty}(G(1, n)) \mid d \lambda\left(V_{J}\right) f=0$, for $\left.J \in T_{3}\right\}$.

\section{REFERENCES}

[G1] F. Gonzalez, Radon transforms on Grassmann manifolds, J. Funct. Anal. 71 (1987), 339362. MR 89a:53081

[G2] F. Gonzalez, Bi-invariant differential operators on the Euclidean motion group and applications to generalized Radon transforms, Ark. Mat. 26 (1988), 191-204. MR 92c:58144

[G3] F. Gonzalez, Invariant differential operators and the range of the Radon D-plane transform, Math. Ann. 287 (1990), 627-635. MR 92a:58141

[G4] F. Gonzalez, On the range of the Radon transform on Grassmann manifolds, preprint.

[GH] F. Gonzalez and S. Helgason, Invariant differential operators on Grassmann manifolds, Adv. in Math. 60 (1986), 81-91. MR 87j:22015

[GK] F. Gonzalez and T. Kakehi, Pfaffian Systems and Radon Transforms on Affine Grassmann Manifolds, Math. Ann. 326 (2003), 237-273.

[Gr] E. Grinberg, Radon transforms on higher rank Grassmannians, J. Diff. Geom. 24 (1986), 53-68. MR 87 m:22023

[GrRu] E. Grinberg and B. Rubin, Radon inversion on Grassmannians via Garding-Gindikin fractional integrals, to appear in Annals of Mathematics.

[GR] I. S. Gradshteyn and I. M. Ryzhik, Table of Integrals, Series, and Products, Academic Press, 1980.

[H1] S. Helgason, The Radon transform on Euclidean spaces, two-point homogeneous spaces, and Grassmann manifolds, Acta Math. 113 (1965), 153-180. MR 30:2530

[H2] S. Helgason, Groups and Geometric Analysis, Academic Press, Orlando, 1984. MR 86c: 22017

[H3] S. Helgason, Geometric Analysis on Symmetric Spaces, AMS, Mathematical Surveys and Monographs, 39 Providence, 1994. MR 96h:43009

[H4] S. Helgason, The Radon Transform, Second edition, Progress in Mathematics, 5, Birkhäuser, Boston, 1999. MR 2000m:44003

[J] F. John, The ultrahyperbolic differential equation with 4 independent variables, Duke Math. J. 4 (1938), 300-322.

[K] T. Kakehi, Integral geometry on Grassmann manifolds and calculus of invariant differential operators, J. Funct. Anal. 168 (1999), 1-45. MR 2000k:53069]

[KN] S. Kobayashi and K. Nomizu, Foundations of differential geometry I, II, Wiley, New York, 1963 and 1969. MR 38:6501

[P] E.E. Petrov, The Radon transform in spaces of matrices and in Grassmann manifolds, Dokl. Akad. Nauk SSSR, 177 (1967), 1504-1507. MR 36:7095

[Ri] F. Richter, On the $k$-dimensional Radon transform of rapidly decreasing functions, Lecture Notes in Math. No. 1209, Berlin, New York, 1986. MR 88a:53071

[Ru1] B. Rubin, Inversion and characterization of the hemispherical transform, J. D'Analyse Math. 77 (1999), 105-128. MR 2001m:44004

[Ru2] B. Rubin, Radon transforms on affine Grassmannians, The Hebrew University of Jerusalem, (2003) preprint.

[So] D. Solmon, Asymptotic formulas for the dual Radon transform, Math. Zeitschr. 195 (1987), 321-343. MR 88i:44006

[St] R. Strichartz, Harmonic analysis on Grassmann bundles, Trans. Amer. Math. Soc. 296 (1986), 387-409. MR 88b:43006

Department of Mathematics, Tufts University, Medford, Massachusetts 02155-7049

E-mail address: fulton.gonzalez@tufts.edu

Institute of Mathematics, University of Tsukuba, Ibaraki, Japan 305-8571

E-mail address: kakehi@math.tsukuba.ac.jp 